\title{
A ROBUST FINITE ELEMENT METHOD FOR NONHOMOGENEOUS DIRICHLET PROBLEMS IN DOMAINS WITH CURVED BOUNDARIES
}

\author{
JAMES H. BRAMBLE AND J. THOMAS KING
}

\begin{abstract}
In this paper we consider a simple finite element method on an approximate polygonal domain using linear elements. The Dirichlet data are transferred in a natural way and the resulting linear system can be solved using multigrid techniques. Our analysis takes into account the change in domain and data transfer, and optimal-error estimates are obtained that are robust in the regularity of the boundary data provided they are at least square integrable. It is proved that the natural extension of our finite element approximation to the original domain is optimal-order accurate.
\end{abstract}

\section{INTRODUCTION}

During the past twenty years there has been considerable interest in finite element methods wherein a given elliptic problem on a domain $\Omega \subset \mathbf{R}^{2}$ is solved approximately on a convenient nearby domain. In most cases the approximate domain is taken to be a piecewise smooth domain whose boundary is made up of polynomial curved segments. The simplest and, in many ways, the most convenient case is to replace $\Omega$ by a polygonal domain. In any event it is then necessary to estimate the effect of domain perturbation. Such estimates were obtained in the early seventies by Strang [23], Strang and Berger [24], Berger, Scott, and Strang [2], Blair [3], and Thomée [25, 26] in the case of homogeneous Dirichlet data.

For nonhomogeneous Dirichlet boundary data there has been little work using standard finite elements. Bramble, Dupont, and Thomée [5] investigated a method on approximating polygonal domains using subspaces of piecewise polynomials of degree greater than two in a modified Nitsche [21] method. The results in [5] are optimal for smooth solutions but no estimates are obtained for rough data. Moreover, the method in [5] is somewhat difficult to implement. More recently, Choudury and Lasiecka [10] show that the standard Nitsche method is robust on a domain with smooth boundary.

Received by the editor December 14, 1992.

1991 Mathematics Subject Classification. Primary 65N30; Secondary 65F10.

This manuscript has been authored under contract number DE-AC02-76CH00016 with the U.S. Department of Energy. Accordingly, the U.S. Government retains a nonexclusive, royalty-free license to publish or reproduce the published form of this contribution, or allow others to do so, for U.S. Government purposes. This work was also supported in part under the National Science Foundation Grant No. DMS-9007185 and by the U.S. Army Research Office through the Mathematical Sciences Institute, Cornell University, Contract No. DAAL03-91-C-0027. 
In the special case where $\Omega$ is a convex polygon (and hence $\Omega=\Omega_{h}$ ) our method coincides with the method introduced by Fix, Gunzburger, and Peterson [15]. The results in [15], for the boundary data $g \in H^{r-1 / 2}(\Gamma)$ with $1 \leq r$, provide optimal $L_{2}$ but suboptimal $H^{1}$ error estimates. Subsequently, French and King [16, 17] obtained optimal-order error estimates $\left(L_{2}\right.$ and $\left.H^{1}\right)$ for this method, and in particular the method is shown to be robust in the regularity of the boundary data. By this we mean that the best possible order of accuracy is attained for rough as well as for smooth boundary data.

For other methods dealing with approximation of the domain by use of curved elements we refer the reader to Ciarlet [11], Ciarlet and Raviart [12], Scott [22], Zlámal [29, 30], Ženíšek [28], and the references contained therein.

In this paper we consider a simple finite element method on an approximate polygonal domain using linear elements. The Dirichlet data are transferred in a natural way and the resulting linear system can be solved using multigrid techniques (cf. [4]). Our analysis takes into account the change in domain and data transfer, and the optimal-order error estimates which are obtained show that the method is robust in the regularity of the boundary data. This aspect is significant for boundary control problems in which rough boundary data may arise (cf. Lasiecka [19]).

Suppose $\Omega \subset \mathbf{R}^{2}$ is a bounded domain whose boundary $\Gamma$ is smooth, say of class $C^{\infty}$. (We will remark at the end on the piecewise smooth case.) Let $x^{(1)}, \ldots, x^{(N)}$ denote $N$ quasiuniformly spaced points on $\Gamma$ with $x^{(N+1)}=$ $x^{(1)}$. Let $\Omega_{h}$ be the polygonal domain with vertices $x^{(1)}, \ldots, x^{(N)}$ and $\Gamma_{h}^{(j)}$ the "half open" edges from $x^{(j)}$ up to $x^{(j+1)}$. Similarly, $\Gamma^{(j)}$ denotes that part of $\Gamma$ between $x^{(j)}$ and $x^{(j+1)}$. Here, $h$ denotes an upper bound for the length of the longest edge, and $N=N_{h}$ is the number of boundary edges. For $h$ sufficiently small the maximum distance between $\Gamma$ and $\Gamma_{h}$, the boundary of $\Omega_{h}$, satisfies

$$
d\left(\Gamma, \Gamma_{h}\right) \leq C h^{2},
$$

where $d\left(\Gamma, \Gamma_{h}\right)=\max _{x \in \Gamma_{h}}\{|x+t \nu|: x+t \nu \in \Gamma\}$ and $\nu$ denotes the unit outward normal to $\Gamma_{h}$. We assume that the length $h_{j}$ of $\Gamma_{h}^{(j)}$ satisfies $\kappa h \leq$ $h_{j} \leq h$, where $\kappa$ is independent of $h$.

Consider the Dirichlet problem on $\Omega$ :

$$
A u=f \text { in } \Omega, \quad u=g \text { on } \Gamma,
$$

where $A$ is the uniformly positive definite second-order elliptic operator

$$
A=-\sum_{i, j=1}^{2} \frac{\partial}{\partial x_{j}}\left(a_{i j}(x) \frac{\partial}{\partial x_{i}}\right)
$$

with smooth coefficients $a_{i j} \in C^{2}\left(\Omega^{\prime}\right)$ with $\bar{\Omega} \subset \Omega^{\prime}$. For this problem we consider a finite element method in which the domain $\Omega$ is replaced by $\Omega_{h} \subset$ $\Omega^{\prime}$, the Dirichlet data $g$ are transferred as $g_{h}$ to $\Gamma_{h}$ by some means, and $f$ is taken to be zero outside of $\Omega$. We determine an approximation $u_{h}$ to $u$ obtained by a simple finite element method, using linear elements, applied to the perturbed problem

$$
A U=f \text { in } \Omega_{h}, \quad U=g_{h} \text { on } \Gamma_{h} .
$$


In this paper we examine a natural choice of $g_{h}$ and derive error estimates in ( $L_{2}$-based) Sobolev spaces. We summarize our main results in the following.

Theorem 1. Let $u_{h}$ be the finite element approximation defined by (3.1). There exists $C$, independent of $h$, such that

$$
\left\|u-u_{h}\right\|_{0, \Omega} \leq C\left(h^{2}\|f\|_{0, \Omega}+h^{s+1 / 2}|g|_{s, \Gamma}\right), \quad 0 \leq s \leq 3 / 2,
$$

and

$$
\left\|u-u_{h}\right\|_{1, \Omega} \leq C\left(h\|f\|_{0, \Omega}+h^{s-1 / 2}|g|_{s, \Gamma}\right), \quad 1 / 2 \leq s \leq 3 / 2,
$$

where the above norms are defined in $\S 2$.

Remark. It should be noted that all computations in our method are carried out on an approximate polygonal domain, $\Omega_{h}$, and the natural extension to $\Omega$ of our approximate solution satisfies the optimal error estimate given in Theorem 1. At the end of the paper we briefly discuss how the fast multigrid techniques of [6] can be applied to solve the system of linear equations resulting from our method.

Throughout this paper, $C$ will denote a generic constant which will always be independent of the mesh parameter $h$ and the functions involved. Sometimes we specifically mention this independence for emphasis.

We now give a brief outline of the paper. In $\S 2$ the relevant function spaces are introduced and some perturbation estimates are proved. The approximate problem is defined in $\S 3$, and the main results are proved there.

\section{FUNCTION SPACES AND PRELIMINARY ESTIMATES}

For a domain $D$ in $\mathbf{R}^{2}$ we denote by $H^{k}(D)$ the usual Sobolev space of integer order $k \geq 0$ with norm $\|\cdot\|_{k, D}$ and seminorm $|\cdot|_{k, D}$ involving only the highest derivatives. The inner products on $L_{2}(\Omega)=H^{0}(\Omega)$ and $L_{2}\left(\Omega_{h}\right)=$ $H^{0}\left(\Omega_{h}\right)$ are given by

$$
(v, w)=\int_{\Omega} v(x) w(x) d x \quad \text { and } \quad(v, w)_{h}=\int_{\Omega_{h}} v(x) w(x) d x .
$$

Let $H^{k}(\Gamma)$ denote the Sobolev space of integer order $k \geq 0$ on $\Gamma$ with norm denoted by $|\cdot|_{k, \Gamma}$. The inner products on $L_{2}(\Gamma)$ and $L_{2}\left(\Gamma_{h}\right)$ are given by

$$
\langle v, w\rangle=\int_{\Gamma} v w d s \quad \text { and } \quad\langle v, w\rangle_{h}=\int_{\Gamma_{h}} v w d s .
$$

For real $r \geq 0$, the spaces $H^{r}(\Omega)$ and $H^{r}(\Gamma)$ are defined by interpolation (cf. Lions and Magenes [20]). As usual, $H_{0}^{1}(\Omega)$ and $H_{0}^{1}\left(\Omega_{h}\right)$ denote the Sobolev spaces of order one whose elements have zero trace on $\Gamma$ and $\Gamma_{h}$, respectively. We define the space $H^{-1 / 2}(\Gamma)$ as the dual of $H^{1 / 2}(\Gamma)$. The norm on $H^{-1 / 2}(\Gamma)$ is given by

$$
|v|_{-1 / 2, \Gamma}=\sup _{\psi \in H^{1 / 2}(\Gamma)} \frac{\langle v, \psi\rangle}{|\psi|_{1 / 2, \Gamma}}
$$

Associated with the elliptic operator $A$ is the bilinear form

$$
a(v, w)=\sum_{i, j=1}^{2} \int_{\Omega} a_{i j}(x) \frac{\partial v}{\partial x_{i}} \frac{\partial w}{\partial x_{j}} d x, \quad v, w \in H^{1}(\Omega),
$$


and $a_{h}(\cdot, \cdot)$ denotes the corresponding form on $\Omega_{h}$. Note that if $\Omega$ is convex, then $\Omega_{h} \subset \Omega$, but in general $\Omega_{h} \not \subset \Omega$. For $f \in L_{2}(\Omega)$ and $g \in H^{r-1 / 2}(\Gamma)$ with $1 \leq r \leq 2$ the solution $u$ of $(1.1)$ is defined in the usual way by

$$
a(u, \phi)=(f, \phi), \quad \phi \in H_{0}^{1}(\Omega) \quad \text { and } \quad u=g \text { on } \Gamma \text {. }
$$

It is well known (cf. Lions and Magenes [20]) that $u \in H^{r}(\Omega)$ and the following elliptic estimate holds:

$$
\|u\|_{r, \Omega} \leq C\left\{\|f\|_{0, \Omega}+|g|_{r-1 / 2, \Gamma}\right\}, \quad 0 \leq r \leq 2 .
$$

For $f \in L_{2}(\Omega)$ and $g \in H^{r-1 / 2}(\Gamma)$, with $0 \leq r<1$, a generalized (very weak) solution of $(1.1)$ is defined by approximation using (2.2). More precisely, let $\left\{g_{n}\right\}$ be a sequence of smooth functions converging to $g$ in $H^{r-1 / 2}(\Gamma)$ and let $\left\{u_{n}\right\}$ denote the corresponding sequence of solutions. Because of $(2.2),\left\{u_{n}\right\}$ is a Cauchy sequence. Its limit, $u \in H^{r}(\Omega)$, is defined to be the weak solution of (1.1).

We want to estimate the effect of domain perturbation and data transfer on our finite element approximation. We begin by defining a natural means of transferring Dirichlet data on $\Gamma$ to $\Gamma_{h}$. Denote the unit outward normal to $\Gamma_{h}^{(j)}$ by $\nu^{(j)}$ and let $x_{h}(t)$ be the parametrization of $\Gamma_{h}^{(j)}$ by arc length. This induces the following parametrization on $\Gamma^{(j)}$ :

$$
X_{h}(t)=x_{h}(t)+\delta_{x_{h}(t)} \nu^{(j)}
$$

where $\left|\delta_{x_{h}(t)}\right|$ is the distance between $x_{h}(t)$ and $\Gamma$ along $\nu^{(j)}$. We assume that $h$ is small enough that $X_{h}(t)$ is well defined. Then define, for a given function $g$ on $\Gamma$,

$$
\tilde{g}\left(x_{h}(t)\right)=g\left(X_{h}(t)\right), \quad x_{h}(t) \in \Gamma_{h}^{(j)},
$$

and note that $\tilde{g}(x)=g(x)$ for $x=x^{(j)}$ or $x=x^{(j+1)}$. The inverse mapping is also well defined. Indeed, there are constants $c$ and $C$, independent of $h$, such that

$$
c|g|_{0, \Gamma} \leq|\tilde{g}|_{0, \Gamma_{h}} \leq C|g|_{0, \Gamma} .
$$

In several places in the analysis in this paper we need to use a bounded linear extension operator (cf. Lions and Magenes [20] and Grisvard [18]) $E$ : $H^{r}(\Omega) \mapsto H^{r}\left(\mathbf{R}^{2}\right)$, with $0 \leq r \leq 2$, satisfying $\left.E \phi\right|_{\Omega}=\phi$ for $\phi \in H^{r}(\Omega)$ and

$$
\|E \phi\|_{r, \mathbf{R}^{2}} \leq C\|\phi\|_{r, \Omega} \text {. }
$$

For an arbitrary function $w \in H^{r}(\Omega)$, with $0 \leq r \leq 2$, we shall make the convention that $w$ has been extended to all of $\overline{\mathbf{R}^{2}}$ by $E$ and, with a slight abuse of notation, we also call the extended function $w$.

We shall need some estimates for functions on the region between $\Omega$ and $\Omega_{h}$.

Let $\Omega_{h}^{(j)}$ be a typical region bounded by $\Gamma^{(j)}$ and $\Gamma_{h}^{(j)}$. Without loss of generality we may assume that $\Gamma_{h}^{(j)}$ has its left endpoint at the origin and is given by $\Gamma_{h}^{(j)}=\left\{(x, y) \mid y=0,0 \leq x<C_{1} h\right\}$ and $\Gamma^{(j)}=\left\{(x, y) \mid y=\delta_{x} \geq\right.$ $\left.0,0 \leq x<C_{1} h\right\}$. Now $\delta_{x} \leq C_{2} h^{2}$ and $\left|\delta_{x}^{\prime}\right| \leq C_{3} h$. It is easy to see, using the divergence theorem that

$$
\int_{\Gamma^{(j)}} \varphi^{2}\left(1+\left(\delta_{x}^{\prime}\right)^{2}\right)^{-1 / 2} d s-\int_{\Gamma_{h}^{(j)}} \varphi^{2} d x=2 \int_{\Omega_{h}^{(j)}} \varphi \frac{\partial \varphi}{\partial y} d x d y
$$


and

$$
\int_{\Omega_{h}^{(j)}} \varphi^{2} d x d y=\int_{\Gamma^{(j)}} y \varphi^{2}\left(1+\left(\delta_{x}^{\prime}\right)^{2}\right)^{-1 / 2} d s-2 \int_{\Omega_{h}^{(j)}} y \varphi \frac{\partial \varphi}{\partial y} d x d y .
$$

It follows from the Cauchy-Schwarz inequality that

$$
\begin{aligned}
& \|\varphi\|_{0, \Omega_{h}^{(j)}}^{2} \leq C\left(h^{2}|\varphi|_{0, \Gamma^{(j)}}^{2}+h^{4}\left\|\frac{\partial \varphi}{\partial y}\right\|_{0, \Omega_{h}^{(j)}}^{2}\right), \\
& \|\varphi\|_{0, \Omega_{h}^{(j)}}^{2} \leq C\left(h^{2}|\varphi|_{0, \Gamma_{h}^{(j)}}^{2}+h^{4}\left\|\frac{\partial \varphi}{\partial y}\right\|_{0, \Omega_{h}^{(j)}}^{2}\right),
\end{aligned}
$$

and

$$
|\psi|_{0, \Gamma^{(j)}}^{2} \leq C h^{2}\left\|\frac{\partial \psi}{\partial y}\right\|_{0, \Omega_{h}^{(j)}}^{2} \quad \text { if } \quad \psi=0 \text { on } \Gamma_{h}^{(j)} .
$$

We can now prove some lemmas which will be used later.

Lemma 1. Suppose $w \in H^{r}(\Omega)$ (extended by $E$ ) with $r \geq 1$ and $\gamma w=g$ denotes the trace of $w$ on $\Gamma$. Then there is a constant $C$, independent of $h$ and $w$, such that

$$
|w-\tilde{g}|_{0, \Gamma_{h}} \leq C h^{r}\|w\|_{r, \Omega}, \quad 1 \leq r \leq 2 .
$$

Proof. We consider $\Omega_{h}^{(j)}$ and apply (2.11) with $\psi=w(x, y)-w(x, 0)$ to obtain

$$
|w-\tilde{g}|_{0, \Gamma_{h}^{(j)}}^{2}=\int_{\Gamma_{h}^{(j)}}|\tilde{\psi}|^{2} d x \leq C|\psi|_{0, \Gamma^{(j)}}^{2} \leq C h^{2}\left\|\frac{\partial w}{\partial y}\right\|_{0, \Omega_{h}^{(j)}}^{2} .
$$

Applying (2.9) to $\frac{\partial w}{\partial y}$, we see that

$$
|w-\tilde{g}|_{0, \Gamma_{h}^{(j)}}^{2} \leq C\left(h^{4}\left|\frac{\partial w}{\partial y}\right|_{0, \Gamma^{(j)}}^{2}+h^{6}\|w\|_{2, \Omega_{h}^{(j)}}^{2}\right) .
$$

Summing over $j$ and using the trace inequality

$$
\sum_{i=1}^{2}\left|\partial w / \partial x_{i}\right|_{0, \Gamma}^{2} \leq C\|w\|_{2, \Omega}^{2}
$$

yields the lemma for $r=2$. Similarly, summing over $j$ in (2.13) proves the lemma for $r=1$, and the result follows by interpolation.

The next two lemmas follow immediately from (2.9) and (2.10), summing over the appropriate indices $j$.

Lemma 2. Suppose that $w \in H^{1}(\Omega)$ (extended by $E$ ). Then there is a constant $C$, independent of $h$ and $w$, such that

$$
\|w\|_{0,\left(\Omega_{h} \backslash \Omega\right) \cup\left(\Omega \backslash \Omega_{h}\right)}^{2} \leq C\left(h^{2}|w|_{0, \Gamma}^{2}+h^{4}|w|_{1, \Omega}^{2}\right) .
$$

Lemma 3. Suppose that $w \in H^{1}\left(\Omega_{h}\right)$. Then there is a constant $C$, independent of $h$ and $w$, such that

$$
\|w\|_{0, \Omega_{h} \backslash \Omega}^{2} \leq C\left(h^{2}|w|_{0, \Gamma_{h}}^{2}+h^{4}|w|_{1, \Omega_{h}}^{2}\right) .
$$


For the purpose of defining and analyzing our finite element method we now define certain spaces of piecewise polynomial functions on $\Gamma$ and $\Gamma_{h}$. Let $S_{h}\left(\Gamma_{h}\right)$ consist of piecewise linear functions of $t$ on $\Gamma_{h}$, continuous on $\Gamma_{h}$ and linear on each edge $\Gamma_{h}^{(j)}$. The space $S_{h}(\Gamma)$ is defined to be all functions of the form

$$
\hat{\chi}\left(X_{h}(t)\right)=\chi\left(x_{h}(t)\right),
$$

where $\chi \in S_{h}\left(\Gamma_{h}\right)$. We define the orthogonal projectors $Q_{h}: L_{2}\left(\Gamma_{h}\right) \mapsto S_{h}\left(\Gamma_{h}\right)$ and $\hat{Q}_{h}: L_{2}(\Gamma) \mapsto S_{h}(\Gamma)$ as follows:

$$
\left\langle Q_{h} g, \chi\right\rangle_{h}=\langle g, \chi\rangle_{h} \text { for all } \chi \in S_{h}\left(\Gamma_{h}\right)
$$

and

$$
\left\langle\hat{Q}_{h} g, \chi\right\rangle=\langle g, \chi\rangle \text { for all } \chi \in S_{h}(\Gamma) .
$$

Now $S_{h}(\Gamma)$ is the space of continuous piecewise linear (with respect to the parameter $t$ ) functions on $\Gamma$. That is, $\varphi \in S_{h}(\Gamma)$ if $\varphi$ is continuous and on each arc, $\Gamma^{(j)}$, is a polynomial of degree less than or equal to one in $t$. The space $S_{h}(\Gamma)$ satisfies the following approximation property. For $q \in H^{r}(\Gamma)$

$$
\inf _{\varphi \in S_{h}(\Gamma)}\left(|q-\varphi|_{0, \Gamma}+h|q-\varphi|_{1, \Gamma)} \leq C h^{r}|q|_{r, \Gamma}, \quad 1 \leq r \leq 2 .\right.
$$

From (2.17) we get

$$
\left|\left(I-\hat{Q}_{h}\right) q\right|_{0, \Gamma}=\inf _{\varphi \in S_{h}(\Gamma)}|q-\varphi|_{0, \Gamma} \leq C h^{2}|q|_{2, \Gamma} .
$$

We also have the trivial estimate

$$
\left|\left(I-\hat{Q}_{h}\right) q\right|_{0, \Gamma} \leq|q|_{0, \Gamma}
$$

It follows, by interpolation between (2.18) and (2.19), that

$$
\left|\left(I-\hat{Q}_{h}\right) q\right|_{0, \Gamma} \leq C h^{s}|q|_{s, \Gamma}, \quad 0 \leq s \leq 2 .
$$

It also follows from (2.17) that

$$
\inf _{\varphi \in S_{h}(\Gamma)}\left(|q-\varphi|_{0, \Gamma}+h^{1 / 2}|q-\varphi|_{1 / 2, \Gamma)} \leq C h^{r}|q|_{r, \Gamma}, \quad 1 \leq r \leq 2 .\right.
$$

The inverse property

$$
|\varphi|_{1, \Gamma} \leq C h^{-1}|\varphi|_{0, \Gamma}, \quad \varphi \in S_{h}(\Gamma),
$$

is standard. It follows by interpolation that, for $0 \leq s \leq 1$,

$$
|\varphi|_{s, \Gamma} \leq C h^{-s}|\varphi|_{0, \Gamma}, \quad \varphi \in S_{h}(\Gamma) .
$$

Finally, using (2.20), (2.21), and (2.22), we can easily show that

$$
\left|\left(I-\hat{Q}_{h}\right) q\right|_{1 / 2, \Gamma} \leq C h^{s-1 / 2}|q|_{s, \Gamma}, \quad 1 / 2 \leq s \leq 2,
$$

and, using the definition of $H^{-1 / 2}(\Gamma)$, that

$$
\left|\left(I-\hat{Q}_{h}\right) q\right|_{-1 / 2, \Gamma} \leq C h^{s+1 / 2}|q|_{s, \Gamma}, \quad 0 \leq s \leq 2 .
$$

The following perturbation estimate will be of importance in our analysis. 
Lemma 4. Let $g \in L_{2}(\Gamma)$. Then

$$
\left|Q_{h} \tilde{g}-\widetilde{\hat{Q}_{h}} g\right|_{0, \Gamma_{h}} \leq C h^{2}|g|_{0, \Gamma},
$$

where $C$ is independent of $h$.

Proof. With $t$ the arc length parameter on $\Gamma_{h}$ and $J(t)$ the Jacobian of the piecewise smooth parametrization $t \mapsto X_{h}(t)$, we have, for $\varphi$ on $\Gamma$,

$$
\int_{\Gamma} \varphi d s=\int_{\Gamma_{h}} \tilde{\varphi} J d t
$$

Note that $J(t)$ is smooth except at the nodes. Also, it is easy to see that if $\varphi \in S_{h}(\Gamma)$, then $\tilde{\varphi} \in S_{h}\left(\Gamma_{h}\right)$, and if $\phi \in S_{h}\left(\Gamma_{h}\right)$, then there is a function $\chi \in S_{h}(\Gamma)$ such that $\phi=\tilde{\chi}$. Now let $\tilde{\chi}=Q_{h} \tilde{g}-\widehat{\hat{Q}_{h}} g$. Then, since $\chi \in S_{h}(\Gamma)$,

$$
\begin{aligned}
\left|Q_{h} \tilde{g}-\widetilde{\hat{Q}_{h} g}\right|_{0, \Gamma_{h}}^{2} & =\int_{\Gamma_{h}}\left(Q_{h} \tilde{g}-\widetilde{\hat{Q}_{h} g}\right) \tilde{\chi} d t=\int_{\Gamma_{h}} \tilde{g} \tilde{\chi} d t-\widetilde{\int_{\Gamma_{h}}} \widetilde{\hat{Q}_{h} g} \tilde{\chi} d t \\
& =\int_{\Gamma} g \chi d s-\int_{\Gamma} \hat{Q}_{h} g \chi d s+\int_{\Gamma_{h}}\left(\tilde{g}-\widetilde{\hat{Q}_{h} g}\right) \tilde{\chi}(1-J) d t \\
& =\int_{\Gamma_{h}}\left(\tilde{g}-\widetilde{\hat{Q}_{h}} g\right) \tilde{\chi}(1-J) d t
\end{aligned}
$$

Using (2.25) and the Cauchy-Schwarz inequality, we get

$$
\left|Q_{h} \tilde{g}-\widetilde{\hat{Q}_{h} g}\right|_{0, \Gamma_{h}}^{2} \leq C\left(\max _{t \in\left[0, l_{h}\right]}|1-J(t)|\right)\left|\tilde{g}-\widetilde{\hat{Q}_{h} g}\right|_{0, \Gamma_{h}}\left|Q_{h} \tilde{g}-\widetilde{\hat{Q}_{h} g}\right|_{0, \Gamma_{h}},
$$

where $l_{h}$ is the length of $\Gamma_{h}$. It follows easily from the definition of $\Gamma_{h}$ that $\max _{t \in\left[0, l_{h}\right]}|1-J(t)| \leq C h^{2}$. Hence we obtain

$$
\left|Q_{h} \tilde{g}-\widetilde{\hat{Q}_{h} g}\right|_{0, \Gamma_{h}} \leq C h^{2}\left|\tilde{g}-\widetilde{\hat{Q}_{h} g}\right|_{0, \Gamma_{h}} \text {. }
$$

Using (2.5), we have

$$
\left|Q_{h} \tilde{g}-\widetilde{\hat{Q}_{h} g}\right|_{0, \Gamma_{h}} \leq C h^{2}\left|g-\hat{Q}_{h} g\right|_{0, \Gamma} \leq C h^{2}|g|_{0, \Gamma},
$$

which is Lemma 4.

We will also need to introduce another boundary space for our analysis. Let $S_{h}^{1}(\Gamma)$ be the space of functions which are cubic polynomials with respect to arc length on each $\Gamma^{(j)}$ and which are continuously differentiable. Define the orthogonal projector $Q_{h}^{1}: L_{2}(\Gamma) \mapsto S_{h}^{1}(\Gamma)$ as follows:

$$
\left\langle Q_{h}^{1} g, \chi\right\rangle=\langle g, \chi\rangle \text { for all } \chi \in S_{h}^{1}(\Gamma) \text {. }
$$

It is well known that $S_{h}^{1}(\Gamma)$ is a subspace of $H^{2}(\Gamma)$ and that the following inverse, approximation, and boundedness properties are satisfied. For $0 \leq s \leq 2$ and $\varphi \in S_{h}^{1}(\Gamma)$,

$$
\begin{gathered}
|\varphi|_{s, \Gamma} \leq C h^{-s}|\varphi|_{0, \Gamma}, \quad \varphi \in S_{h}^{1}(\Gamma), \\
\left|\left(I-Q_{h}^{1}\right) q\right|_{-1 / 2, \Gamma}+h^{1 / 2}\left|\left(I-Q_{h}^{1}\right) q\right|_{0, \Gamma} \leq C h^{s+1 / 2}|q|_{s, \Gamma}
\end{gathered}
$$

and

$$
\left|Q_{h}^{1} q\right|_{s, \Gamma} \leq C|q|_{s, \Gamma} .
$$

These inequalities are easy generalizations of results in [9]. 


\section{THE FINITE ELEMENT METHOD}

We define the approximate boundary data on $\Gamma_{h}$ by

$$
g_{h}=Q_{h} \tilde{g}
$$

We assume the family of polygonal domains, $\left\{\Omega_{h}\right\}$, and corresponding family of triangulations, $\left\{\mathscr{T}_{h}\right\}$, satisfy the usual sort of quasiuniformity condition. The only vertices on $\Gamma_{h}$ of a triangle $\tau_{h} \in \mathscr{T}_{h}$ are vertices of $\Gamma_{h}$, and every triangle $\tau_{h} \in \mathscr{T}_{h}$ is affine equivalent to a reference triangle. Define the space $V_{h}$ to consist of continuous piecewise linear functions relative to the triangulation $\mathscr{T}_{h}$. The boundary space $V_{h}\left(\Gamma_{h}\right)$ denotes the restriction of $V_{h}$ to $\Gamma_{h}$ and coincides with $S_{h}\left(\Gamma_{h}\right)$.

The Approximate Problem. The approximate solution $u_{h} \in H^{1}(\Omega)$ of (1.1) is defined as follows: In $\Omega_{h}$ let $u_{h} \in V_{h}$ be the solution of

$$
a_{h}\left(u_{h}, \phi\right)=(f, \phi)_{h}, \quad \phi \in V_{h}^{0}, \quad \text { and } \quad u_{h}=g_{h} \text { on } \Gamma_{h},
$$

where $V_{h}^{0}=V_{h} \cap H_{0}^{1}\left(\Omega_{h}\right)$ and $f=0$ outside $\Omega$. Further define $u_{h}$ in $\Omega \backslash \Omega_{h}$ as follows: In $\Omega_{h}^{(j)} \not \subset \Omega_{h}$ with $\tau_{h}^{(j)}$ the triangle in $\Omega_{h}$ having $\Gamma_{h}^{(j)}$ as one of its sides, $u_{h}$ is the linear extension from $\tau_{h}^{(j)}$ to $\Omega_{h}^{(j)}$.

Note that this definition of $u_{h}$ outside the region $\Omega_{h}$ is the most natural one and $u_{h}$ is thus defined in all of $\Omega$.

As a consequence of the quasiuniformity assumptions, the space $V_{h}$ has the following simultaneous approximation property. For $w \in H^{r}\left(\Omega_{h}\right), 1 \leq r \leq 2$, there is a function $w_{h} \in V_{h}$ such that

$$
\left\|w-w_{h}\right\|_{0, \Omega_{h}}+h\left\|w-w_{h}\right\|_{1, \Omega_{h}} \leq C h^{r}\|w\|_{r, \Omega_{h}},
$$

where $C$ is independent of $h$ and $w$. This property can be established using a trianglewise argument that is given in Bramble and $\mathrm{Xu}$ [9].

The following result will be needed in our analysis.

Lemma 5. Let $w \in H^{2}(\Omega)$ and $\phi_{h} \in V_{h}$. Then

$$
\inf _{\chi \in V_{h}^{0}}\left\{\left\|w-\phi_{h}-\chi\right\|_{0, \Omega_{h}}+h\left\|w-\phi_{h}-\chi\right\|_{1, \Omega_{h}}\right\} \leq C\left(h^{2}\|w\|_{2, \Omega_{h}}+h^{1 / 2}\left|w-\phi_{h}\right|_{0, \Gamma_{h}}\right) .
$$

Proof. Let $w_{h} \in V_{h}$ satisfy (3.2). Choose $\chi \in V_{h}^{0}$ equal to $w_{h}-\phi_{h}$ at the interior nodes of $\Omega_{h}$. Then, a straightforward calculation yields

$$
\begin{aligned}
\left\|w_{h}-\phi_{h}-\chi\right\|_{0, \Omega_{h}}+h\left\|w_{h}-\phi_{h}-\chi\right\|_{1, \Omega_{h}} & \leq C h\left(\sum_{j=1}^{N}\left|w_{h}\left(x^{(j)}\right)-\phi_{h}\left(x^{(j)}\right)\right|^{2}\right)^{1 / 2} \\
& \leq C h^{1 / 2}\left|w_{h}-\phi_{h}\right|_{0, \Gamma_{h}} .
\end{aligned}
$$

The last inequality follows since $w_{h}-\phi_{h} \in S_{h}\left(\Gamma_{h}\right)$ and on $S_{h}\left(\Gamma_{h}\right)$ the norms on the right above are equivalent. Thus, using the triangle inequality, we have

$$
\begin{aligned}
& \inf _{\chi \in V_{h}^{0}}\left\{\left\|w-\phi_{h}-\chi\right\|_{0, \Omega_{h}}+h\left\|w-\phi_{h}-\chi\right\|_{1, \Omega_{h}}\right\} \\
& \quad \leq\left\|w-w_{h}\right\|_{0, \Omega_{h}}+h\left\|w-w_{h}\right\|_{1, \Omega_{h}}+C h^{1 / 2}\left|w_{h}-\phi_{h}\right|_{0, \Gamma_{h}} .
\end{aligned}
$$


Moreover (see Dupont [14]), for $v \in H^{1}\left(\Omega_{h}\right)$, the following estimate holds

$$
|v|_{0, \Gamma_{h}}^{2} \leq C\|v\|_{0, \Omega_{h}}\|v\|_{1, \Omega_{h}},
$$

where $C$ is independent of $h$. Consequently, taking $v=w-w_{h}$, we get

$$
h^{1 / 2}\left|w-w_{h}\right|_{0, \Gamma_{h}} \leq C\left(\left\|w-w_{h}\right\|_{0, \Omega_{h}}+h\left\|w-w_{h}\right\|_{1, \Omega_{h}}\right) .
$$

Hence, using (3.3), the triangle inequality and this inequality, we obtain

$$
\begin{aligned}
& \inf _{\chi \in V_{h}^{0}}\left\{\left\|w-\phi_{h}-\chi\right\|_{0, \Omega_{h}}+h\left\|w-\phi_{h}-\chi\right\|_{1, \Omega_{h}}\right\} \\
& \quad \leq\left\|w-w_{h}\right\|_{0, \Omega_{h}}+h\left\|w-w_{h}\right\|_{1, \Omega_{h}}+C h^{1 / 2}\left|w-\phi_{h}\right|_{0, \Gamma_{h}} .
\end{aligned}
$$

The lemma now follows from this and (3.2).

We will need to know that functions in $H_{0}^{1}(\Omega)$ can be approximated well by functions in $V_{h}^{0}$. This is provided in the following lemma.

Lemma 6. Let $w \in H^{2}(\Omega) \cap H_{0}^{1}(\Omega)$ (extended by $E$ ). Then

$$
\inf _{\chi \in V_{h}^{0}}\left\{\|w-\chi\|_{0, \Omega_{h}}+h\|w-\chi\|_{1, \Omega_{h}}\right\} \leq C h^{2}\|w\|_{2, \Omega} .
$$

Proof. Take $\phi_{h}=0$ and $w \in H_{0}^{1}(\Omega)$ in Lemma 5. Then

$$
\inf _{\chi \in V_{h}^{0}}\left\{\|w-\chi\|_{0, \Omega_{h}}+h\|w-\chi\|_{1, \Omega_{h}}\right\} \leq C\left(h^{2}\|w\|_{2, \Omega}+h^{1 / 2}|w|_{0, \Gamma_{h}}\right) .
$$

It follows from Lemma 1 that

$$
|w|_{0, \Gamma_{h}} \leq C h^{2}\|w\|_{2, \Omega}
$$

Combining the last two inequalities proves Lemma 6.

Our goal is to prove Theorem 1 with $u_{h}$ defined by (3.1). To this end, we will consider separately the cases $f=0$ and $g=0$, and let $u_{h, 0}$ be defined analogously to $u_{h}$ in (3.1) but with $g_{h}=0$. Define $u_{0}$ to be the solution of

$$
A u_{0}=f \text { in } \Omega, \quad u_{0}=0 \text { on } \Gamma \text {. }
$$

We now prove the following.

Proposition 1. There exists a constant $C$, independent of $h$, such that

$$
\left\|u_{0}-u_{h, 0}\right\|_{i, \Omega_{h}} \leq C h^{2-i}\|f\|_{0, \Omega}, \quad i=0,1 .
$$

Proof. Without loss, because of ellipticity, we may take the seminorm to be $|\cdot|_{1, \Omega_{h}}^{2}=a_{h}(\cdot, \cdot)$. For $\chi \in V_{h}^{0}$,

$$
\left|u_{0}-u_{h, 0}\right|_{1, \Omega_{h}}^{2}=a_{h}\left(u_{0}-u_{h, 0}, u_{0}-\chi\right)+a_{h}\left(u_{0}-u_{h, 0}, \chi-u_{h, 0}\right) .
$$

Now, since $\chi-u_{h, 0} \in V_{h}^{0}$,

$$
\begin{aligned}
a_{h}\left(u_{0}-u_{h, 0}, \chi-u_{h, 0}\right) & =\left(A u_{0}, \chi-u_{h, 0}\right)_{h}-\left(f, \chi-u_{h, 0}\right)_{h} \\
& =\int_{\Omega_{h} \backslash \Omega} A u_{0}\left(\chi-u_{h, 0}\right) d x \\
& \leq C\left\|u_{0}\right\|_{2, \Omega}\left\|\chi-u_{h, 0}\right\|_{0, \Omega_{h} \backslash \Omega .}
\end{aligned}
$$

By (2.2) and Lemma 3,

$$
a_{h}\left(u_{0}-u_{h, 0}, \chi-u_{h, 0}\right) \leq C h^{2}\|f\|_{0, \Omega}\left|\chi-u_{h, 0}\right|_{1, \Omega_{h}} .
$$


This inequality, together with (3.5) and the Cauchy-Schwarz inequality, yield for any $\chi \in V_{h}^{0}$

$$
\left|u_{0}-u_{h, 0}\right|_{1, \Omega_{h}} \leq C\left(\left|u_{0}-\chi\right|_{1, \Omega_{h}}+h^{2}\|f\|_{0, \Omega}\right) .
$$

Using Lemma 6 and (2.2), we obtain

$$
\left|u_{0}-u_{h, 0}\right|_{1, \Omega_{h}} \leq C h\|f\|_{0, \Omega} .
$$

We next estimate $\left\|u_{0}-u_{h, 0}\right\|_{0, \Omega_{h}}$ by using a duality argument. To this end, let $\varphi \in C_{0}^{\infty}\left(\Omega_{h}\right)$ and define $w \in H^{2}(\Omega) \cap H_{0}^{1}(\Omega)$ to be the solution of

$$
A w=\varphi \text { in } \Omega, \quad w=0 \text { on } \Gamma
$$

and extended by $E$. Then for $\chi \in V_{h}^{0}$

$$
\begin{aligned}
\left(u_{0}-u_{h, 0}, \varphi\right)_{h}= & \left(u_{0}-u_{h, 0}, A w\right)_{h}+\left(u_{0}-u_{h, 0}, \varphi-A w\right)_{h} \\
= & a_{h}\left(u_{0}-u_{h, 0}, w-\chi\right)+a_{h}\left(u_{0}-u_{h, 0}, \chi\right) \\
& -\left\langle u_{0}, \partial w / \partial \nu_{h}\right\rangle_{h}+\left(u_{0}-u_{h, 0}, \varphi-A w\right)_{h},
\end{aligned}
$$

where $\partial w / \partial \nu_{h}$ is the outward conormal derivative defined almost everywhere on $\Gamma_{h}$. Now we have

$$
\begin{aligned}
a_{h}\left(u_{0}\right. & \left.-u_{h, 0}, w-\chi\right)+a_{h}\left(u_{0}-u_{h, 0}, \chi\right) \\
& \leq\left|u_{0}-u_{h, 0}\right|_{1, \Omega_{h}}|w-\chi|_{1, \Omega_{h}}+\left(A u_{0}-f, \chi\right)_{h} \\
& \leq\left|u_{0}-u_{h, 0}\right|_{1, \Omega_{h}}|w-\chi|_{1, \Omega_{h}}+C\|f\|_{0, \Omega}\|\chi\|_{0, \Omega_{h} \backslash \Omega} \\
& \leq\left(\left|u_{0}-u_{h, 0}\right|_{1, \Omega_{h}}+C h^{2}\|f\|_{0, \Omega}\right)|w-\chi|_{1, \Omega_{h}}+C h^{2}\|f\|_{0, \Omega}\|w\|_{1, \Omega},
\end{aligned}
$$

where the last inequality follows by the triangle inequality and Lemma 3 . Using Lemma 6 (with $\chi$ taken to be the minimizing function) and (2.2), we get

$$
\begin{aligned}
& a_{h}\left(u_{0}-u_{h, 0}, w-\chi\right)+a_{h}\left(u_{0}-u_{h, 0}, \chi\right) \\
& \quad \leq C\left(h\left|u_{0}-u_{h, 0}\right|_{1, \Omega_{h}}+h^{2}\|f\|_{0, \Omega}\right)\|\varphi\|_{0, \Omega_{h}} .
\end{aligned}
$$

We also have, using Lemma 1 and (2.2),

$$
\begin{aligned}
\left\langle u_{0}, \partial w / \partial \nu_{h}\right\rangle_{h} & \leq\left|u_{0}\right|_{0, \Gamma_{h}}\left|\partial w / \partial \nu_{h}\right|_{0, \Gamma_{h}} \\
& \leq C h^{2}\left\|u_{0}\right\|_{2, \Omega}\|w\|_{2, \Omega} \\
& \leq C h^{2}\|f\|_{0, \Omega}\|\varphi\|_{0, \Omega_{h} .}
\end{aligned}
$$

Here we used the inequality (see Lemma 8 of [5])

$$
\left|\partial w / \partial \nu_{h}\right|_{0, \Gamma_{h}} \leq C\|w\|_{2, \Omega},
$$

with $C$ independent of $h$. This inequality is also easily derived using (2.7), (2.8), and (2.14). Finally, using the definition of $w$ and the Cauchy-Schwarz inequality, we obtain

$$
\left(u_{0}-u_{h, 0}, \varphi-A w\right)_{h} \leq C\left\|u_{0}-u_{h, 0}\right\|_{0, \Omega_{h} \backslash \Omega}\left(\|w\|_{2, \mathbf{R}^{2}}+\|\varphi\|_{0, \Omega_{h}}\right) .
$$

By Lemma 3,

$$
\left(u_{0}-u_{h, 0}, \varphi-A w\right)_{h} \leq C\left(h\left|u_{0}\right|_{0, \Gamma_{h}}+h^{2}\left|u_{0}-u_{h, 0}\right|_{1, \Omega_{h}}\right)\|\varphi\|_{0, \Omega_{h}} .
$$

Thus, by Lemma 1 and (2.2),

$$
\left(u_{0}-u_{h, 0}, \varphi-A w\right)_{h} \leq C\left(h^{3}\|f\|_{0, \Omega}+h^{2}\left|u_{0}-u_{h, 0}\right|_{1, \Omega_{h}}\right)\|\varphi\|_{0, \Omega_{h}} .
$$


Combining (3.8), (3.9), (3.10), and (3.12), we have

$$
\frac{\left(u_{0}-u_{h, 0}, \varphi\right)_{h}}{\|\varphi\|_{0, \Omega_{h}}} \leq C\left(h\left|u_{0}-u_{h, 0}\right|_{1, \Omega_{h}}+h^{2}\|f\|_{0, \Omega}\right) .
$$

Thus, using (3.6), we have

$$
\left\|u_{0}-u_{h, 0}\right\|_{0, \Omega_{h}}=\sup _{\varphi \in C_{0}^{\infty}\left(\Omega_{h}\right)} \frac{\left(u_{0}-u_{h, 0}, \varphi\right)_{h}}{\|\varphi\|_{0, \Omega_{h}}} \leq C h^{2}\|f\|_{0, \Omega} .
$$

This proves Proposition 1.

We now consider the case $f=0$. We handle this case by an indirect analysis where we introduce another finite element approximation, $v_{h}$. Specifically, let $v_{h}$ be defined as in (3.1) but with $f=0$ and $v_{h}=\widehat{\hat{Q}_{h} g}$ on $\Gamma_{h}$. We further define $\hat{u}^{h}$ and $u^{h}$ to be the $A$-harmonic functions satisfying

$$
\hat{u}^{h}=\hat{Q}_{h} g, \quad u^{h}=Q_{h}^{1} g, \quad \text { on } \Gamma \text {. }
$$

Then, since $Q_{h}^{1} g \in H^{3 / 2}(\Gamma)$, it follows from (2.2) that $u^{h} \in H^{2}(\Omega)$. This, as we will see, is the reason for introducing $S_{h}^{1}(\Gamma) \subset H^{2}(\Gamma)$. We prove that $v_{h}$ is an optimal approximation to $u-u_{0}$. First we need the following estimate.

Proposition 2. There exists a constant $C$, independent of $h$, such that

$$
\left\|\hat{u}^{h}-v_{h}\right\|_{0, \Omega_{h}} \leq C h^{s+1 / 2}|g|_{s, \Gamma}, \quad 0 \leq s \leq 3 / 2,
$$

and

$$
\left\|\hat{u}^{h}-v_{h}\right\|_{1, \Omega_{h}} \leq C h^{r-1 / 2}|g|_{r, \Gamma}, \quad 1 / 2 \leq r \leq 3 / 2,
$$

where we recall that, by convention, $\hat{u}^{h}$ is defined in $\Omega_{h}$ by $E \hat{u}^{h}$.

Proof. The analysis in what follows is similar to that of Proposition 1, however the differences are significant. We first note that it suffices to prove (3.14) with $\hat{u}^{h}$ replaced by $u^{h}$ since, by (2.6), (2.2), and properties of $Q_{h}^{1}$ and $\hat{Q}_{h}$,

$$
\left\|\hat{u}^{h}-u^{h}\right\|_{1, \Omega_{h}} \leq\left\|E\left(\hat{u}^{h}-u^{h}\right)\right\|_{1, \mathbf{R}^{2}} \leq C\left\|\hat{u}^{h}-u^{h}\right\|_{1, \Omega} \leq h^{r-1 / 2}|g|_{r, \Gamma}
$$

for $1 / 2 \leq r \leq 3 / 2$. We first consider the seminorm. Then, for $\chi \in V_{h}^{0}$,

$$
\left|u^{h}-v_{h}\right|_{1, \Omega_{h}}^{2}=a_{h}\left(u^{h}-v_{h}, u^{h}-v_{h}-\chi\right)+a_{h}\left(u^{h}-v_{h}, \chi\right) .
$$

Now, since $\chi \in V_{h}^{0}$,

$$
a_{h}\left(u^{h}-v_{h}, \chi\right)=\left(A u^{h}, \chi\right)_{h}=\int_{\Omega_{h} \backslash \Omega} A u^{h} \chi d x \leq C\left\|u^{h}\right\|_{2, \Omega}\|\chi\|_{0, \Omega_{h} \backslash \Omega} .
$$

By (2.2) and Lemma 3,

$$
\begin{aligned}
a_{h}\left(u^{h}-v_{h}, \chi\right) & \leq C h^{2}\left|Q_{h}^{1} g\right|_{3 / 2, \Gamma}|\chi|_{1, \Omega_{h}} \\
& \leq C h^{2}\left|Q_{h}^{1} g\right|_{3 / 2, \Gamma}\left(\left|u^{h}-v_{h}-\chi\right|_{1, \Omega_{h}}+\left|u^{h}-v_{h}\right|_{1, \Omega_{h}}\right) .
\end{aligned}
$$

This inequality, together with (3.15) and the Cauchy-Schwarz inequality, yield for any $\chi \in V_{h}^{0}$

$$
\left|u^{h}-v_{h}\right|_{1, \Omega_{h}} \leq C\left(\left|u^{h}-v_{h}-\chi\right|_{1, \Omega_{h}}+h^{2}\left|Q_{h}^{1} g\right|_{3 / 2, \Gamma}\right) .
$$


Using Lemma 5 and (2.2), we obtai..

$$
\left|u^{h}-v_{h}\right|_{1, \Omega_{h}} \leq C\left(h\left|Q_{h}^{1} g\right|_{3 / 2, \Gamma}+h^{-1 / 2}\left|u^{h}-v_{h}\right|_{0, \Gamma_{h}}\right) .
$$

But, from the definitions of $u^{h}$ and $v_{h}$,

$$
\left|u^{h}-v_{h}\right|_{0, \Gamma_{h}} \leq\left|u^{h}-\widetilde{Q_{h}^{1} g}\right|_{0, \Gamma_{h}}+\left|\widetilde{Q_{h}^{1} g}-\widetilde{\hat{Q}_{h} g}\right|_{0, \Gamma_{h}} .
$$

Using Lemma 1, (2.2), and (2.5), we conclude that

$$
\left|u^{h}-v_{h}\right|_{1, \Omega_{h}} \leq C\left(h\left|Q_{h}^{1} g\right|_{3 / 2, \Gamma}+h^{-1 / 2}\left|Q_{h}^{1} g-\hat{Q}_{h} g\right|_{0, \Gamma}\right) .
$$

From the properties of $Q_{h}^{1}$ and $\hat{Q}_{h}$ we obtain

$$
\left|\hat{u}^{h}-v_{h}\right|_{1, \Omega_{h}} \leq C h^{r-1 / 2}|g|_{r, \Gamma}, \quad 1 / 2 \leq r \leq 3 / 2 .
$$

This proves (3.14) for the seminorm.

We next estimate $\left\|\hat{u}^{h}-v_{h}\right\|_{0, \Omega_{h}}$ by using a duality argument. This time, we estimate $\hat{u}^{h}-v_{h}$ directly, but, even so, $u^{h}$ plays a role in the analysis. To this end, let $\varphi \in C_{0}^{\infty}\left(\Omega_{h}\right)$ and define $w \in H^{2}(\Omega) \cap H_{0}^{1}(\Omega)$ as in (3.7). Then for $\chi \in V_{h}^{0}$

$$
\begin{aligned}
\left(\hat{u}^{h}-v_{h}, \varphi\right)_{h}= & \left(\hat{u}^{h}-v_{h}, A w\right)_{h}+\left(\hat{u}^{h}-v_{h}, \varphi-A w\right)_{h} \\
= & a_{h}\left(\hat{u}^{h}-v_{h}, w-\chi\right)+a_{h}\left(\hat{u}^{h}-v_{h}, \chi\right) \\
& -\left\langle\hat{u}^{h}-v_{h}, \partial w / \partial \nu_{h}\right\rangle_{h}+\left(\hat{u}^{h}-v_{h}, \varphi-A w\right)_{h} .
\end{aligned}
$$

Now

$$
\begin{aligned}
a_{h}\left(\hat{u}^{h}\right. & \left.-v_{h}, w-\chi\right)+a_{h}\left(\hat{u}^{h}-v_{h}, \chi\right) \\
& \leq\left|\hat{u}^{h}-v_{h}\right|_{1, \Omega_{h}}|w-\chi|_{1, \Omega_{h}}+\left(A u^{h}, \chi\right)_{h}+a_{h}\left(\hat{u}^{h}-u^{h}, \chi\right) \\
& \leq\left|\hat{u}^{h}-v_{h}\right|_{1, \Omega_{h}}|w-\chi|_{1, \Omega_{h}}+C\left\|u^{h}\right\|_{2, \Omega}\|\chi\|_{0, \Omega_{h} \backslash \Omega}+a_{h}\left(\hat{u}^{h}-u^{h}, \chi\right) \\
& \leq\left(\left|\hat{u}^{h}-v_{h}\right|_{1, \Omega_{h}}+C h^{2}\left|Q_{h}^{1} g\right|_{3 / 2, \Gamma)|w-\chi|_{1, \Omega_{h}}}\right. \\
& +C h^{2}\left|Q_{h}^{1} g\right|_{3 / 2, \Gamma}\|w\|_{1, \Omega}+a_{h}\left(\hat{u}^{h}-u^{h}, \chi\right) .
\end{aligned}
$$

In order to estimate the last term in (3.18), recalling that $w \in H_{0}^{1}(\Omega)$, let $\bar{w}$ denote $w$ extended by zero outside of $\Omega$. Similarly, extend $\chi$ by zero outside $\Omega_{h}$. We then write

$$
a_{h}\left(\hat{u}^{h}-u^{h}, \chi\right)=a_{h}\left(\hat{u}^{h}-u^{h}, \chi-\bar{w}\right)+a_{h}\left(\hat{u}^{h}-u^{h}, \bar{w}\right) .
$$

Then, since $\hat{u}^{h}-u^{h}$ is $A$-harmonic, $a\left(\hat{u}^{h}-u^{h}, \bar{w}\right)=0$ and hence

$$
a_{h}\left(\hat{u}^{h}-u^{h}, \bar{w}\right) \leq C\left|E\left(\hat{u}^{h}-u^{h}\right)\right|_{1, \mathbf{R}^{2}}|\bar{w}|_{1, \Omega \backslash \Omega_{h}} .
$$

Now from the definitions of $w$ and $\bar{w}$

$$
\begin{aligned}
a_{h}\left(\hat{u}^{h}-u^{h}, \chi-\bar{w}\right) & \leq C\left|E\left(\hat{u}^{h}-u^{h}\right)\right|_{1, \mathbf{R}^{2}}|\bar{w}-\bar{\chi}|_{1, \Omega \cup \Omega_{h}} \\
& \leq C\left|E\left(\hat{u}^{h}-u^{h}\right)\right|_{1, \mathbf{R}^{2}}\left(|w-\chi|_{1, \Omega_{h}}+|w|_{1,\left(\Omega_{h} \backslash \Omega\right) \cup\left(\Omega \backslash \Omega_{h}\right)}\right) .
\end{aligned}
$$

Combining the last two inequalities with (3.19), we conclude that

$$
a_{h}\left(\hat{u}^{h}-u^{h}, \chi\right) \leq C\left\|\hat{u}^{h}-u^{h}\right\|_{1, \Omega}\left(|w-\chi|_{1, \Omega_{h}}+|w|_{1,\left(\Omega_{h} \backslash \Omega\right) \cup\left(\Omega \backslash \Omega_{h}\right)}\right) .
$$

Using Lemma 2, (2.2), and (2.14), we have

$$
a_{h}\left(\hat{u}^{h}-u^{h}, \chi\right) \leq C\left|\hat{Q}_{h} g-Q_{h}^{1} g\right|_{1 / 2, \Gamma}\left(|w-\chi|_{1, \Omega_{h}}+h\|w\|_{2, \Omega}\right) .
$$


Combining (3.18) and (3.20) gives

$$
\begin{aligned}
& a_{h}\left(\hat{u}^{h}-v_{h}, w-\chi\right)+a_{h}\left(\hat{u}^{h}-v_{h}, \chi\right) \\
& \leq\left(\left|\hat{u}^{h}-v_{h}\right|_{1, \Omega_{h}}+C h^{2}\left|Q_{h}^{1} g\right|_{3 / 2, \Gamma}+\left|\hat{Q}_{h} g-Q_{h}^{1} g\right|_{1 / 2, \Gamma}\right)|w-\chi|_{1, \Omega_{h}} \\
& \quad+C\left(h^{2}\left|Q_{h}^{1} g\right|_{3 / 2, \Gamma}+h\left|\hat{Q}_{h} g-Q_{h}^{1} g\right|_{1 / 2, \Gamma}\right)\|w\|_{2, \Omega} .
\end{aligned}
$$

Taking $\chi$ to be the minimizing function in Lemma 6 and using (2.2), we have

$$
\begin{aligned}
& a_{h}\left(\hat{u}^{h}-v_{h}, w-\chi\right)+a_{h}\left(\hat{u}^{h}-v_{h}, \chi\right) \\
& \quad \leq\left(h\left|\hat{u}^{h}-v_{h}\right|_{1, \Omega_{h}}+C h^{2}\left|Q_{h}^{1} g\right|_{3 / 2, \Gamma}+h\left|\hat{Q}_{h} g-Q_{h}^{1} g\right|_{1 / 2, \Gamma}\|\varphi\|_{0, \Omega} .\right.
\end{aligned}
$$

We also have, using Lemma 1 (twice), (3.11) and (2.2),

$$
\begin{aligned}
\left\langle\hat{u}^{h}-\right. & \left.v_{h}, \partial w / \partial \nu_{h}\right\rangle_{h} \leq\left|\hat{u}^{h}-v_{h}\right|_{0, \Gamma_{h}}\left|\partial w / \partial \nu_{h}\right|_{0, \Gamma_{h}} \\
& \leq C\left|\hat{u}^{h}-\widetilde{\gamma \hat{u}^{h}}\right|_{0, \Gamma_{h}}\|w\|_{2, \Omega} \\
& \leq C\left(\left|\left(\hat{u}^{h}-u^{h}\right)-\gamma\left(\hat{u}^{h}-u^{h}\right)\right|_{0, \Gamma_{h}}+\left|u^{h}-\widetilde{\gamma u^{h}}\right|_{0, \Gamma_{h}}\right)\|\varphi\|_{0, \Omega} \\
& \leq C\left(h\left\|\hat{u}^{h}-u^{h}\right\|_{1, \Omega}+h^{2}\left\|u^{h}\right\|_{2, \Omega}\right) \mid \varphi \|_{0, \Omega} \\
& \leq C\left(h\left|\hat{Q}_{h} g-Q_{h}^{1} g\right|_{1 / 2, \Gamma}+h^{2}\left|Q_{h}^{1} g\right|_{3 / 2, \Gamma}\right)\|\varphi\|_{0, \Omega} .
\end{aligned}
$$

Finally, we estimate the last term in (3.17). Since $A w=\varphi$ in $\Omega$,

$$
\left(\hat{u}^{h}-v_{h}, \varphi-A w\right)_{h} \leq C\left\|\hat{u}^{h}-v_{h}\right\|_{0, \Omega_{h} \backslash \Omega}\|\varphi\|_{0, \Omega} .
$$

Analogously to (3.22), it follows that

$$
\left(\hat{u}^{h}-v_{h}, \varphi-A w\right)_{h} \leq C\left(h\left|\hat{Q}_{h} g-Q_{h}^{1} g\right|_{1 / 2, \Gamma}+h^{2}\left|Q_{h}^{1} g\right|_{3 / 2, \Gamma}\right)\|\varphi\|_{0, \Omega} .
$$

Combining (3.17), (3.21), (3.22), and (3.23), we conclude that

$$
\begin{aligned}
\left\|\hat{u}^{h}-v_{h}\right\|_{0, \Omega_{h}} & =\sup _{\varphi \in C_{0}^{\infty}\left(\Omega_{h}\right)} \frac{\left(\hat{u}^{h}-v_{h}, \varphi\right)_{h}}{\|\varphi\|_{0, \Omega_{h}}} \\
& \leq C\left(h\left|\hat{Q}_{h} g-Q_{h}^{1} g\right|_{1 / 2, \Gamma}+h^{2}\left|Q_{h}^{1} g\right|_{3 / 2, \Gamma}\right) \\
& \leq C h^{r+1 / 2}|g|_{r, \Gamma}, \quad 0 \leq r \leq 3 / 2 .
\end{aligned}
$$

This completes the proof of Proposition 2.

We next prove the following

Proposition 3. Let $u_{H}$ be the A-harmonic function equal to $g$ on $\Gamma$. Then there exists a constant $C$, independent of $h$, such that

and

$$
\left\|u_{H}-v_{h}\right\|_{0, \Omega_{h}} \leq C h^{s+1 / 2}|g|_{s, \Gamma}, \quad 0 \leq s \leq 3 / 2,
$$

$$
\left\|u_{H}-v_{h}\right\|_{1, \Omega_{h}} \leq C h^{s-1 / 2}|g|_{s, \Gamma}, \quad 1 / 2 \leq s \leq 3 / 2,
$$

where we recall that $u_{H}$ is extended to $\Omega_{h}$ by $E u_{H}$.

Proof. It is clear from Proposition 2 that we only need to estimate $\left\|u_{H}-\hat{u}^{h}\right\|_{i, \Omega_{h}}$ for $i=0$ and 1. But, from (2.2), we have

$$
\begin{aligned}
\left\|u_{H}-\hat{u}^{h}\right\|_{0, \Omega_{h}} & \leq C\left\|E\left(u_{H}-\hat{u}^{h}\right)\right\|_{0, \mathbf{R}^{2}} \leq C\left\|u_{H}-\hat{u}^{h}\right\|_{0, \Omega} \\
& \leq C\left|g-\hat{Q}_{h} g\right|_{-1 / 2, \Gamma} \leq C h^{s+1 / 2}|g|_{s, \Gamma}, \quad 0 \leq s \leq 3 / 2,
\end{aligned}
$$

with the analogous inequality when $i=1$. This, together with Proposition 2 , proves Proposition 3.

We now can prove an estimate for $u-u_{h}$. 
Proposition 4. There exists a constant $C$, independent of $h$, such that

$$
\left\|u-u_{h}\right\|_{0, \Omega_{h}} \leq C\left(h^{2}\|f\|_{0, \Omega}+h^{s+1 / 2}|g|_{s, \Gamma}\right), \quad 0 \leq s \leq 3 / 2,
$$

and

$$
\left\|u-u_{h}\right\|_{1, \Omega_{h}} \leq C\left(h\|f\|_{0, \Omega}+h^{s-1 / 2}|g|_{s, \Gamma}\right), \quad 1 / 2 \leq s \leq 3 / 2 .
$$

Proof. Set $u_{h, H}=u_{h}-u_{h, 0}$. Because of Proposition 1, it remains to estimate $u_{H}-u_{h, H}$. For $i=0$ or 1 ,

$$
\left\|u_{H}-u_{h, H}\right\|_{i, \Omega_{h}} \leq\left\|u_{H}-v_{h}\right\|_{i, \Omega_{h}}+\left\|v_{h}-u_{h, H}\right\|_{1, \Omega_{h}} .
$$

By Lemma 2.7 of Dupont [14] we have, for some constant $C$, independent of $h$,

$$
\left\|v_{h}-u_{h, H}\right\|_{1, \Omega_{h}} \leq C\left(\left|v_{h}-u_{h, H}\right|_{1, \Omega_{h}}+\left|v_{h}-u_{h, H}\right|_{0, \Gamma_{h}}\right) .
$$

Since $v_{h}-u_{h, H}$ is discrete $A$-harmonic, it follows that

$$
\left|v_{h}-u_{h, H}\right|_{1, \Omega_{h}} \leq\left|v_{h}-u_{h, H}-\chi\right|_{1, \Omega_{h}}
$$

for any $\chi \in V_{h}^{0}$. Applying Lemma 5 with $\phi_{h}=u_{h}-v_{h}$ and $w=0$ shows that

$$
\left|v_{h}-u_{h, H}\right|_{1, \Omega_{h}} \leq \inf _{\chi \in V_{h}^{0}}\left|v_{h}-u_{h, H}-\chi\right|_{1, \Omega_{h}} \leq C h^{-1 / 2}\left|v_{h}-u_{h, H}\right|_{0, \Gamma_{h}} .
$$

Thus, using (3.25) and (3.26), we get

$$
\left\|v_{h}-u_{h, H}\right\|_{1, \Omega_{h}} \leq C h^{-1 / 2}\left|v_{h}-u_{h, H}\right|_{0, \Gamma_{h}} .
$$

By the definition of $u_{h, H}$ and $v_{h}$, we have $u_{h, H}-v_{h}=Q_{h} \tilde{g}-\widetilde{\hat{Q}_{h} g}$ on $\Gamma_{h}$. Also, $Q_{h} \widetilde{\hat{Q}_{h} g}=\widetilde{\hat{Q}_{h} g}=\hat{Q}_{h} \widehat{\hat{Q}}_{h} g$. Hence, setting $G=g-\hat{Q}_{h} g$ and using Lemma 4 , we obtain

$$
\left|u_{h, H}-v_{h}\right|_{0, \Gamma_{h}}=\left|Q_{h} \tilde{G}-\widetilde{\hat{Q}_{h} G}\right|_{0, \Gamma_{h}} \leq C h^{2}|G|_{0, \Gamma}=C h^{2}\left|\left(I-\hat{Q}_{h}\right) g\right|_{0, \Gamma}
$$

Combining (3.27), (3.28), and (2.20), we see that

$$
\left\|v_{h}-u_{h, H}\right\|_{1, \Omega_{h}} \leq C h^{s+3 / 2}|g|_{s, \Gamma} \text { for } 0 \leq s \leq 3 / 2 .
$$

This estimate, (3.24) and Proposition 3 together prove Proposition 4.

We now prove Theorem 1. For $i=0$ or 1 ,

$$
\left\|u-u_{h}\right\|_{i, \Omega} \leq\left\|u-u_{h}\right\|_{i, \Omega_{h}}+\left\|u-u_{h}\right\|_{i, \Omega \backslash \Omega_{h}} .
$$

Because of Proposition 4, it is sufficient to estimate $\left\|u-u_{h}\right\|_{i, \Omega \backslash \Omega_{h}}$. To do this, we set $w^{h}=u_{0}+u^{h}$ and note that $w^{h} \in H^{2}(\Omega)$. Recall that, according to our convention, $w^{h}$ also denotes the extension by $E$ to $\mathbf{R}^{2}$. Now

$$
\left\|u-u_{h}\right\|_{i, \Omega \backslash \Omega_{h}} \leq\left\|u-w^{h}\right\|_{i, \Omega}+\left\|w^{h}-u_{h}\right\|_{i, \Omega \backslash \Omega_{h}} .
$$

Since $A\left(u-w^{h}\right)=0$, we have from (2.2) and the properties of $Q_{h}^{1}$,

$$
\left|u-w^{h}\right|_{1, \Omega} \leq C h^{s-1 / 2}|g|_{s, \Gamma}, \quad 1 / 2 \leq s \leq 3 / 2
$$

and

$$
\left\|u-w^{h}\right\|_{0, \Omega} \leq C h^{s+1 / 2}|g|_{s, \Gamma}, \quad 0 \leq s \leq 3 / 2 .
$$


Therefore, it remains to estimate $\left\|w^{h}-u_{h}\right\|_{i, \Omega \backslash \Omega_{h}}$, and we do this as follows. Apply (2.10) to the derivatives of $w^{h}-u_{h}$ and note that $u_{h}$ is linear on $\Omega_{h}^{(j)}$, so that its second derivatives vanish there. Then it follows that

$$
\left|w^{h}-u_{h}\right|_{1, \Omega_{h}^{(j)}}^{2} \leq C\left(h^{2} \sum_{i=1}^{2}\left|\partial / \partial x_{i}\left(w^{h}-u_{h}\right)\right|_{0, \Gamma_{h}^{(j)}}^{2}+h^{4}\left\|w^{h}\right\|_{2, \Omega_{h}^{(j)}}^{2}\right) .
$$

It is easy to show from the divergence theorem that

$$
h|v|_{0, \Gamma_{h}^{(j)}}^{2} \leq C\left(\|v\|_{0, \tau_{h}^{(j)}}^{2}+h^{2}|v|_{1, \tau_{h}^{(j)}}^{2}\right) .
$$

Applying (3.35) to the derivatives of $w^{h}-u_{h}$ in (3.34), we obtain

$$
\left|w^{h}-u_{h}\right|_{1, \Omega_{h}^{(j)}}^{2} \leq C\left(h\left|w^{h}-u_{h}\right|_{1, \tau_{h}^{(j)}}^{2}+h^{3}\left\|w^{h}\right\|_{2, \Omega_{h}^{(j)} \cup \tau_{h}^{(j)}}^{2}\right) .
$$

Summing over the appropriate indices $j$, we have

$$
\left|w^{h}-u_{h}\right|_{1, \Omega \backslash \Omega_{h}} \leq C\left(h^{1 / 2}\left|w^{h}-u_{h}\right|_{1, \Omega_{h}}+h^{3 / 2}\left\|w^{h}\right\|_{2, \Omega}\right) .
$$

Similarly, apply (2.10) to $w^{h}-u_{h}$, use (3.35) with $v=w^{h}-u_{h}$ and sum over the appropriate indices $j$ to obtain

$$
\left\|w^{h}-u_{h}\right\|_{0, \Omega \backslash \Omega_{h}} \leq C\left(h^{1 / 2}\left\|w^{h}-u_{h}\right\|_{0, \Omega_{h}}+h^{3 / 2}\left|w^{h}-u_{h}\right|_{1, \Omega \cup \Omega_{h}}\right) .
$$

Combining (3.37) and (3.36), we have

$$
\left\|w^{h}-u_{h}\right\|_{0, \Omega \backslash \Omega_{h}} \leq C\left(h^{1 / 2}\left\|w^{h}-u_{h}\right\|_{0, \Omega_{h}}+h^{3 / 2}\left|w^{h}-u_{h}\right|_{1, \Omega_{h}}+h^{3}\left\|w^{h}\right\|_{2, \Omega}\right) .
$$

Consequently, applying the triangle inequality in (3.36) and using Proposition 4 and (3.32), we conclude that

$$
\left|w^{h}-u_{h}\right|_{1, \Omega \backslash \Omega_{h}} \leq C h^{1 / 2}\left(h\|f\|_{0, \Omega}+h^{s-1 / 2}|g|_{s, \Gamma}\right), \quad 1 / 2 \leq s \leq 3 / 2 .
$$

We also used here (2.2) and the properties of $Q_{h}^{1}$. Using (3.31), (3.32) and this inequality gives

$$
\left|u-u_{h}\right|_{1, \Omega \backslash \Omega_{h}} \leq C\left(h\|f\|_{0, \Omega}+h^{s-1 / 2}|g|_{s, \Gamma}\right), \quad 1 / 2 \leq s \leq 3 / 2 .
$$

In order to estimate $\left\|w^{h}-u_{h}\right\|_{0, \Omega \backslash \Omega_{h}}$, we use (3.38). From Proposition 4 and (3.33) we see that

$$
h^{1 / 2}\left\|w^{h}-u_{h}\right\|_{0, \Omega_{h}} \leq C h^{1 / 2}\left(h^{2}\|f\|_{0, \Omega}+h^{s+1 / 2}|g|_{s, \Gamma}\right), \quad 0 \leq s \leq 3 / 2 .
$$

By using (2.2) and the properties of $Q_{h}^{1}$, we have

$$
h^{3}\left\|w^{h}\right\|_{2, \Omega} \leq C h\left(h^{2}\|f\|_{0, \Omega}+h^{s+1 / 2}|g|_{s, \Gamma}\right), \quad 0 \leq s \leq 3 / 2 .
$$

In order to estimate the second term on the right of (3.38), we write $w^{h}-u_{h}=$ $\left(u_{0}-u_{h, 0}\right)+\left(u^{h}-v_{h}\right)+\left(v_{h}-u_{h, H}\right)$. Applying Proposition 1 to $\left(u_{0}-u_{h, 0}\right)$, (3.16) to $\left(u^{h}-v_{h}\right)$, and (3.29) to $\left(v_{h}-u_{h, H}\right)$, we conclude that

$$
h^{3 / 2}\left|w^{h}-u_{h}\right|_{1, \Omega_{h}} \leq C h^{1 / 2}\left(h^{2}\|f\|_{0, \Omega}+h^{s+1 / 2}|g|_{s, \Gamma}\right), \quad 0 \leq s \leq 3 / 2 .
$$

Hence, combining (3.40), (3.41) and (3.42), we get

$$
\left\|w^{h}-u_{h}\right\|_{0, \Omega \backslash \Omega_{h}} \leq C h^{1 / 2}\left(h^{2}\|f\|_{0, \Omega}+h^{s+1 / 2}|g|_{s, \Gamma}\right), \quad 0 \leq s \leq 3 / 2 .
$$

Using (3.31), (3.33) and (3.43) gives 


$$
\left\|u-u_{h}\right\|_{0, \Omega \backslash \Omega_{h}} \leq C\left(h^{2}\|f\|_{0, \Omega}+h^{s+1 / 2}|g|_{s, \Gamma}\right), \quad 0 \leq s \leq 3 / 2 .
$$

Theorem 1 now follows from (3.44), (3.39) and Proposition 4.

Remark. There is no difficulty extending all of the above to the case in which the boundary is piecewise smooth, provided that there are a finite number of corner points with interior angles less than or equal to $\pi$. In such a case we require that the corner points be contained in the set of points $\left\{x^{(j)}\right\}$. The requisite regularity results may be found in [13].

Remark. The system of linear equations which arises from our approximate method is not adversely affected by the inhomogeneous boundary data. This simply changes the right-hand side in the matrix equation. In order to apply multigrid techniques, we can develop a set of nested spaces as is done in [6]. One starts with a coarse grid of the type described here and successively refines the mesh, using a halving strategy. New triangles are introduced near the boundary by halving the boundary arcs with respect to the parameter $t$. This process is continued until a sufficiently fine mesh is reached. The space $V_{h}$ is then defined relative to the fine mesh. The set of nested subspaces, for the purpose of defining an efficient multigrid algorithm, is defined as in [6] by taking subspaces of the succesively coarser spaces with elements that vanish on all triangles having vertices on the boundary. The resulting multigrid algorithm is uniformly convergent, independent of the mesh parameter $h$.

We note that our method has the following features: It (1) is relatively simple to implement, (2) is robust in the regularity of the boundary data, (3) provides an optimal-order approximate solution on all of $\Omega$, and (4) the resulting approximate solution is computable using fast multigrid techniques.

\section{BIBLIOGRAPHY}

1. A. Berger, $L_{2}$ error estimates for finite elements with interpolated boundary conditions, Numer. Math. 21 (1973), 345-349.

2. A. Berger, L. R. Scott, and G. Strang, Approximate boundary conditions in the finite element method, Symposia Mathematica, X, Academic Press, New York, 1972, pp. 295-313.

3. J. J . Blair, Bounds for the change in the solutions of second order elliptic PDE's when the boundary is perturbed, SIAM J. Appl. Math. 24 (1973), 277-285.

4. J. H. Bramble, Multigrid methods, Lecture notes, Cornell University, 1992.

5. J. H. Bramble, T. Dupont, and V. Thomée, Projection methods for Dirichlet's problem in approximating polygonal domains with boundary value corrections, Math. Comp. 26 (1972), 869-879.

6. J. H. Bramble and J. E. Pasciak, New estimates for multilevel algorithms including the $V$-cycle, Math. Comp. 60 (1993), 447-471.

7. J. H. Bramble, J. Pasciak, and A. H. Schatz, The construction of preconditioners for elliptic problems by substructuring. I, Math. Comp. 47 (1986), 103-134.

8. J. H. Bramble, J. Pasciak, and J. Xu, The analysis of multigrid algorithms with nonnested spaces or noninherited quadratic forms, Math. Comp. 56 (1991), 1-34.

9. J. H. Bramble and J. Xu, Some estimates for a weighted $L^{2}$ projection, Math. Comp. 56 (1991), 463-476.

10. G. Choudury and I. Lasiecka, Optimal convergence rates for semidiscrete approximations of parabolic problems with nonsmooth boudary data, Numer. Funct. Anal. Optim. 12(4\&5) (1991), 469-485. 
11. P. G. Ciarlet, Basic error estimates for elliptic problems, Handbook of Numerical Analysis, Vol. II (P. G. Ciarlet and J. L. Lions, eds.), North-Holland, Amsterdam, 1991, pp. 17-351.

12. P. G. Ciarlet and P. A. Raviart, The combined effect of curved boundaries and numerical integration in isoparametric finite element methods, The Mathematical Foundations of the Finite Element Method with Applications to Partial Differential Equations (A. K. Aziz, ed.), Academic Press, New York, 1972, pp. 409-474.

13. M. Dauge, Elliptic boundary value problems on corner domains: smoothness and asymptotics of solutions, Lecture Notes in Math., vol. 1341, Springer-Verlag, New York, 1988.

14. T. Dupont, $L_{2}$ error estimates for projecting methods for parabolic equations in approximating domains, Mathematical Aspects of Finite Elements in Partial Differential Equations (C. de Boor, ed.), Academic Press, New York, 1974, pp. 313-352.

15. G. J. Fix, M. D. Gunzburger, and J. S. Peterson, On finite element approximations of problems having inhomogeneous essential boundary conditions, Comput. Math. Appl. 9 (1983), 687-700.

16. D. A. French and J. T. King, Approximation of an elliptic control problem by the finite element method, Numer. Funct. Anal. Optim. 12 (1991), 299-314.

17. __ Analysis of a robust finite element approximation for a parabolic equation with rough boundary data, Math. Comp. 60 (1993), 79-104.

18. P. Grisvard, Elliptic problems in nonsmooth domains, Pitman, London, 1985.

19. I. Lasiecka, Galerkin approximation of abstract parabolic boundary value problems with rough boundary data $-L_{p}$ theory, Math. Comp. 47 (1986), 55-75.

20. J. L. Lions and E. Magenes, Nonhomogeneous boundary value problems and applications. I, Springer-Verlag, Berlin, 1972.

21. J. Nitsche, Lineare Spline-funktionen und die Methoden von Ritz für elliptische Randwertprobleme, Arch. Rational Mech. Anal. 36 (1970), 348-355.

22. L. R. Scott, Interpolated boundary conditions in the finite element method, SIAM J. Numer. Anal. 12 (1975), 404-427.

23. G. Strang, Variational crimes in the finite element method, The Mathematical Foundations of the Finite Element Method with Applications to Partial Differential Equations (A. K. Aziz, ed.), Academic Press, New York, 1972, pp. 689-710.

24. G. Strang and A. E. Berger, The change in solution due to change in domain, Partial Differential Equations (D. C. Spencer, ed.), Proc. Sympos. Pure Math., vol. 23, Amer. Math. Soc., Providence, RI, 1973, pp. 199-205.

25. V. Thomée, Polygonal domain approximation in Dirichlet's problem, J. Inst. Math. Appl. 11 (1973), 33-44.

26. __ Approximate solution of Dirichlet's problem using approximating polygonal domains, Topics in Numerical Analysis (J. J. H. Miller, ed.), Academic Press, New York, 1973, pp. 311-328.

27. J. Xu, Theory of multilevel methods, Thesis, Cornell University, Ithaca, New York, 1989.

28. A. Ženíšek, Nonhomogeneous boundary conditions and curved triangular finite elements, Apl. Mat. 26 (1981), 121-141.

29. M. Zlámal, Curved elements in the finite element method. I, SIAM J. Numer. Anal. 10 (1973), 229-240.

30. __ Curved elements in the finite element method. II, SIAM J. Numer. Anal. 11 (1974), 347-362.

Department of Mathematics, Cornell University, Ithaca, New York 14853

E-mail address: bramble@math.cornell.edu

Department of Mathematical Sciences, University of Cincinnati, Cincinnati, Ohio 45221-0025

E-mail address: kingjt@ucbeh.san.uc.edu 